\title{
Why is gene therapy so attractive and so controversial?
}

\author{
Jacques P. Tremblay, Ph.D.
}

Professor of Molecular Medicine, University of Laval

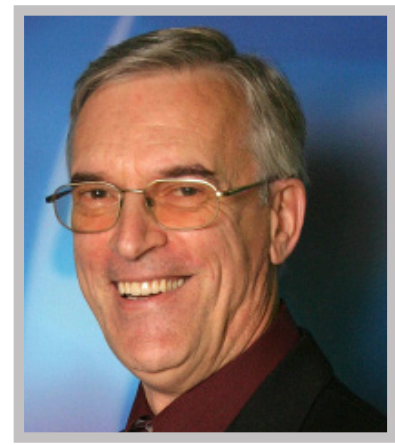

All the characteristics of the human body are encoded in DNA molecules; long double stranded helixes. These DNA molecules are made of only 4 nucleotides: adenosine, cytosine, guanine, and thymidine. It is a sequence of these nucleotides that constitutes a gene that determines, for example, the colour of our eyes or of our hairs. The human genome (i.e., all our genes) contains about 3 billion nucleotides received from our father and 3 billion nucleotides received from our mother. The complete sequence of all these nucleotides has been initially obtained by the collaborative work of hundreds of laboratories over several years and has cost several billion dollars. It is now possible to obtain the complete genome sequence of one individual in one laboratory in one week for less than $\$ 1,000$. All hereditary diseases are due to modifications of the sequence of nucleotides of a gene and the progress in the rapidity of genome sequencing has permitted to identify modifications (i.e., mutations) responsible for 7000 different hereditary diseases.

Now that the modifications responsible for so many hereditary diseases are known, the next logical step is to develop therapies for them. Since these diseases are due to gene modifications, a therapeutic approach that would apply to all of them would be to replace or correct the defective gene - this is gene therapy.

During the last 25 years, gene therapy has been aiming to compensate for a defective gene by introducing a complete copy of the normal gene. This normal gene can be inserted in the patient cells in culture. The genetically corrected cells are then proliferated and transplanted in the appropriate organ of the patient with a genetic disease. This approach can work for some tissues like the muscles, where muscle precursor cells (i.e., myoblasts) can be injected in the muscles and fuse with the existing muscle fibers. However, such a therapeutic approach requiring cell transplantation does not work for complex tissues such as the brain. Thus, an alternative approach is to deliver the normal gene directly to the cells in the human body. This delivery can be achieved via viral vector (i.e., a virus in which the viral genes have been removed and replaced by a therapeutic gene). This gene therapy approach has already been used successfully to treat some diseases, such as Leber amaurosis (an hereditary blindness) and Hemophilia type B (a coagulation problem).

However in the last 5 years, new techniques now permit to correct specifically the defective gene (i.e., replacing the few nucleotides that are missing or incorrect) instead of introducing a complete replacement gene. This new approach is very advantageous when the gene is very big, like for example, the dystrophin gene mutated in Duchenne muscular dystrophy is more than 10 million nucleotides long and thus impossible to include in most viral vectors.

For some persons (usually not those affected by an hereditary disease), gene therapy is controversial because it touches a very fundamental aspect of life. Indeed for these persons, this is as controversial as saying that the universe was not made in 7 days, that the earth is not the centre of the universe, or permitting to transplant a human heart. The capacity to modify genes opens the possibility of curing most, if not all, hereditary diseases. However, as any knowledge, this could also eventually be used to modify other human characteristics not for a medical treatment. Currently, gene therapy is closely supervised by regulatory agencies such as the Food and Drug Administration (FDA) to make sure that the aims are scientifically and ethically correct.

Dr. Tremblay has obtained a PhD in Neurosciences from the University of California in San Diego in 1974. He has been at Laval University in Québec since, as a post-doctoral fellow, a professor and a department chairman. He is currently a full professor in the Department of Molecular Medicine. He has published over 250 scientific articles. 